\title{
ABOUT WORKING OUT OF THE SOCIAL SECURITY INDICATORS IN GEORGIA
}

\section{ELENE CHIKOVANI}

Academic Doctor of Economics, Professor

Tbilisi Open Teaching University, Georgia

elene_customs@yahoo.com

Abstract. Article is devoted to the problems of the working out of the social security indicators in Georgia. It considers the essence of the social security and its importance for crisis and post crisis economies. It evaluates existing deficiencies in working out of the social security indicators in Georgia. It singles out the issues of the necessity and importance of consideration of the security as a compound part of the welfare in the crisis economy. Marking out, the circle of the security indicators related to the development of the basic needs, welfare foundations and capacities.

The necessity of the dissociate of the indicators on the national, family and individual (personal) levels is substantiated. Inevitability of the setting out of the new circle of the social equality indicators in Georgia is grounded. The existing situation in the informational provision of the main directions of the social security recommended by the conventions and guidelines of the international labor organization is characterized.

Special attention is paid to the existing backdowns in development of the food security information system in Georgia. The interrelation of the food security to the problems of the health, food insufficiency of the complex and sustainable development, as well as environmental issues is pointed out. The problems of the evaluation of the food security situation and elaboration of the relevant policy in Georgia are accentuated. The necessity of the further measures towards the improvement of the informational provision of the food security system is substantiated.

KEYWORDS: SOCIAL SECURITY, INDICATOR, INFORMATION PROVISION.

For citation: Chikovani, E., (2020). About Working out of the Social Security Indicators in Georgia. Globalization and Business, 10. 86-93. (In Georgian) https://doi.org/10.35945/gb.2020.10.011 


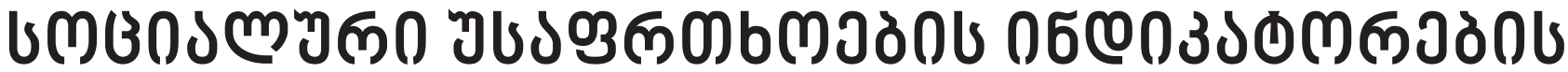

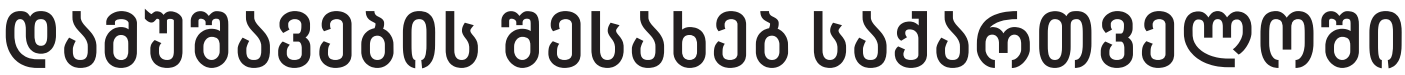

\section{วМอธอ โОภМЗЈธก}

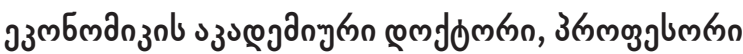

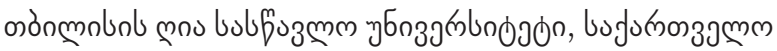

elene_customs@yahoo.com

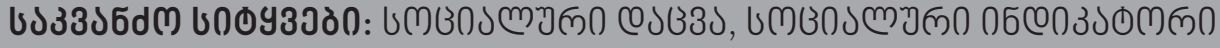

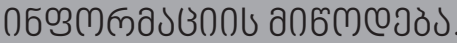

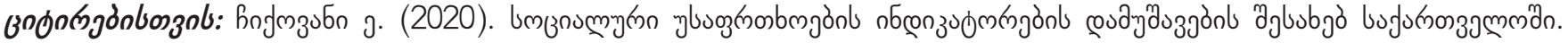

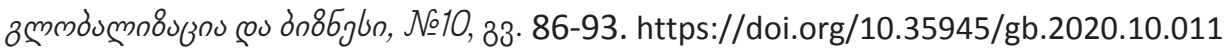

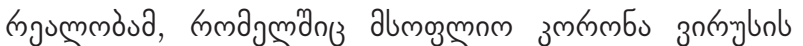

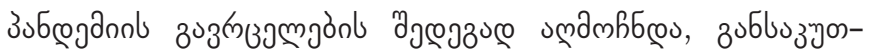

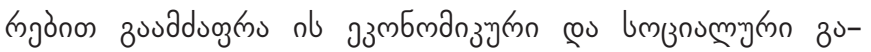

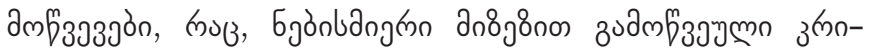

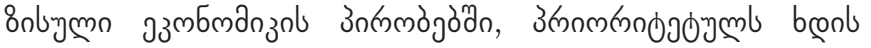

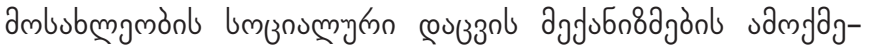

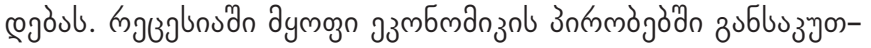

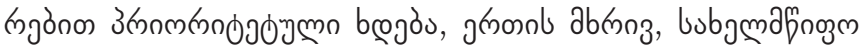

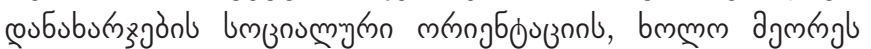

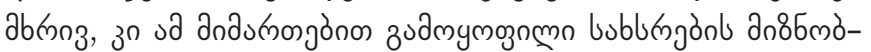

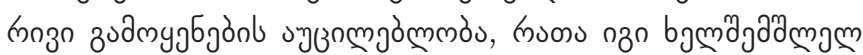

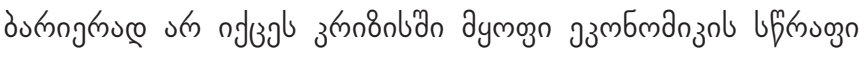

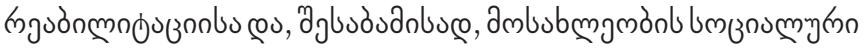

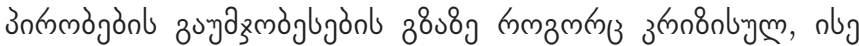

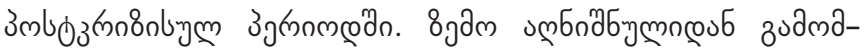

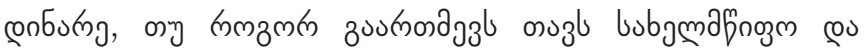

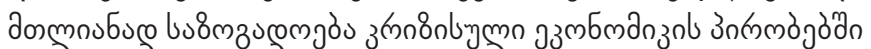

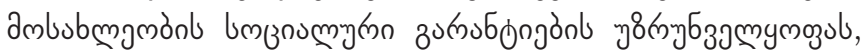

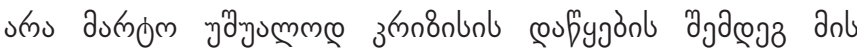

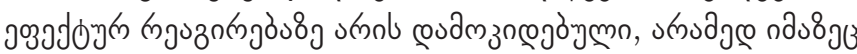

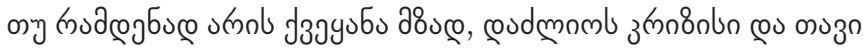

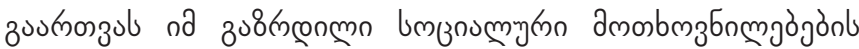

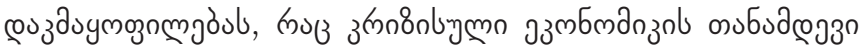

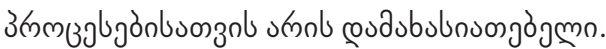

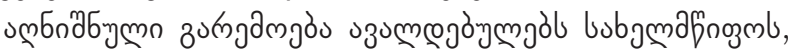

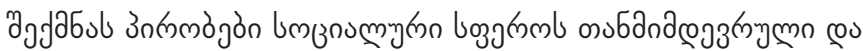

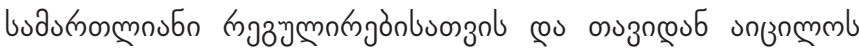

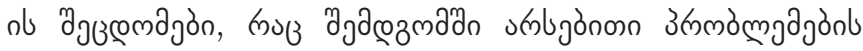

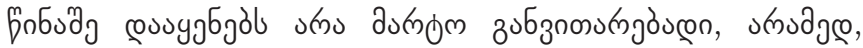

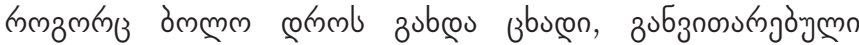

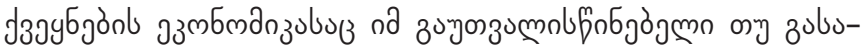

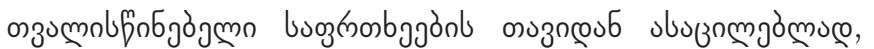

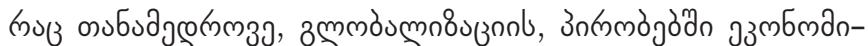

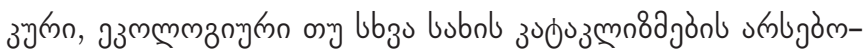

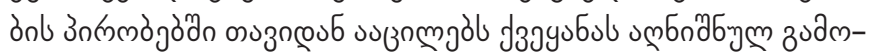

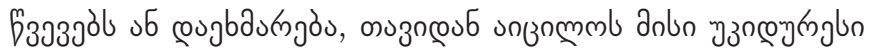

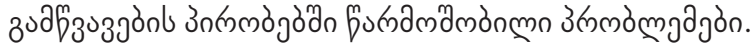

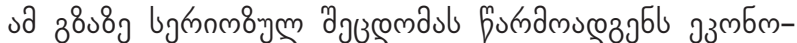

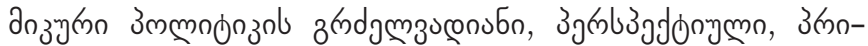

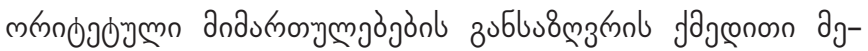

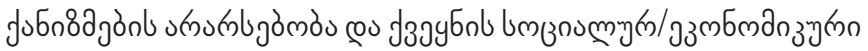

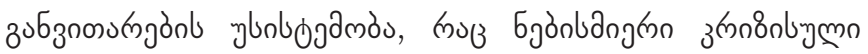

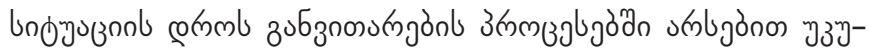

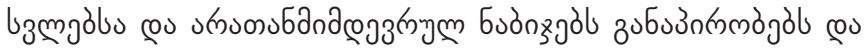

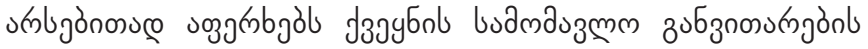

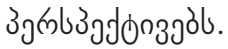

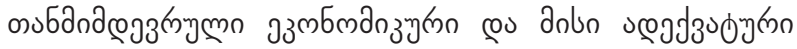

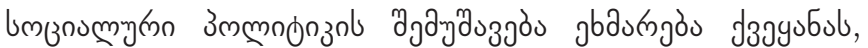

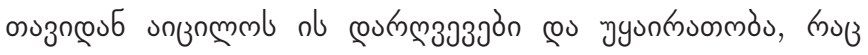

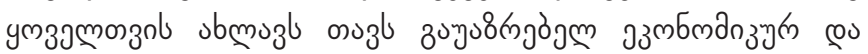

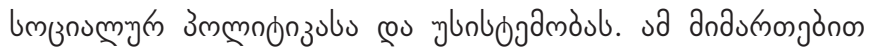

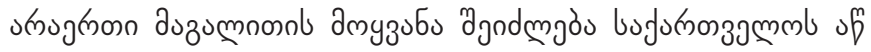

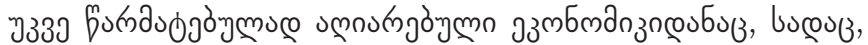

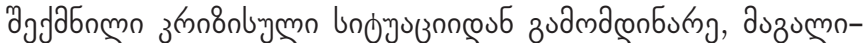

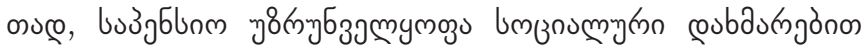
s

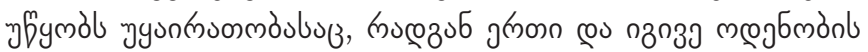

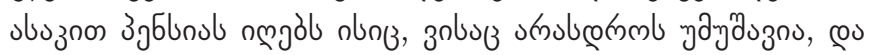

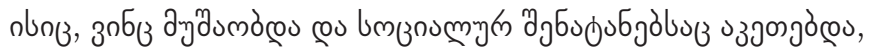

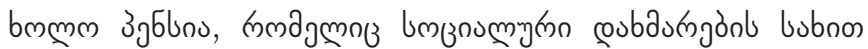

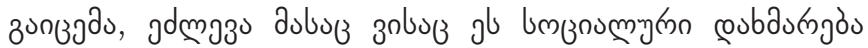

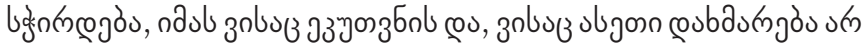

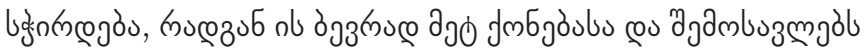

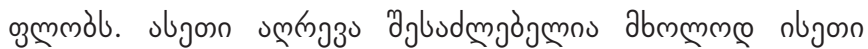




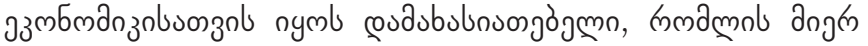

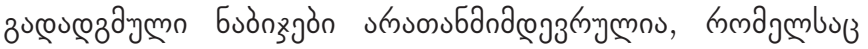

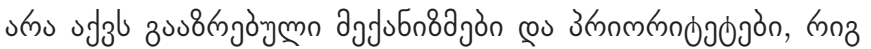

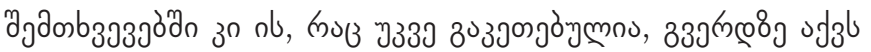

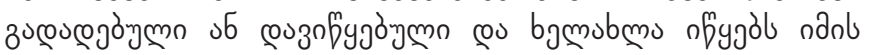

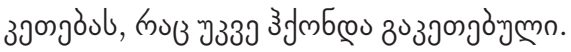

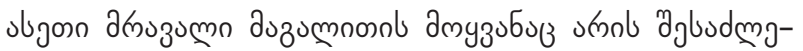

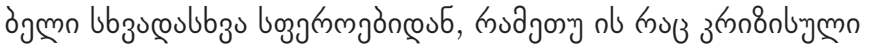

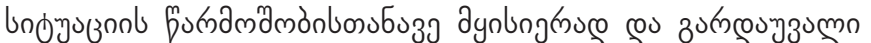

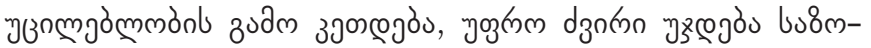

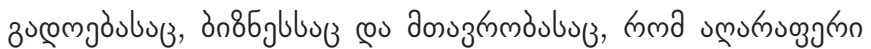

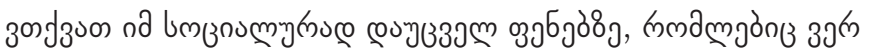

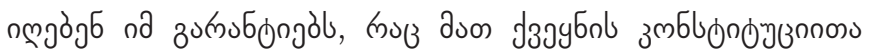

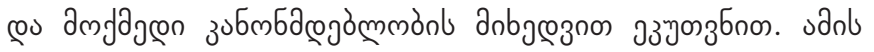

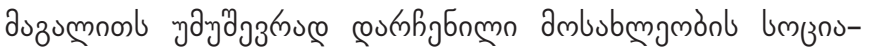

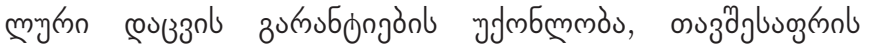

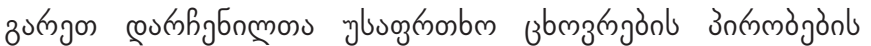

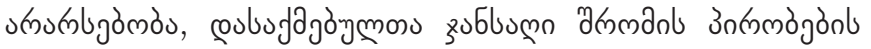

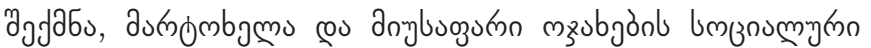
च8

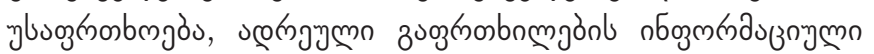

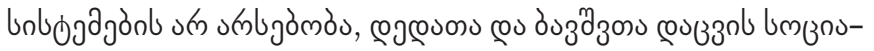

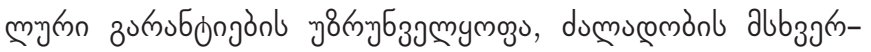

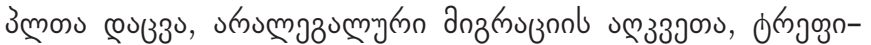

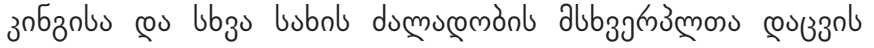

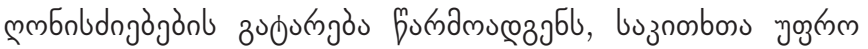

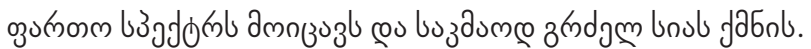

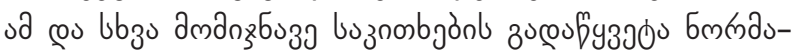

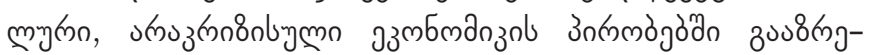

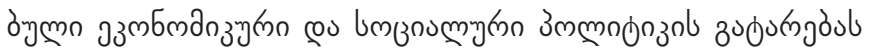

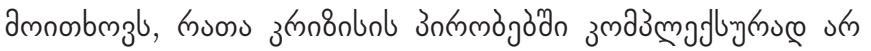

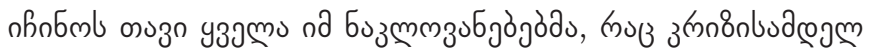

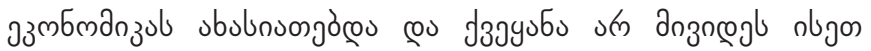

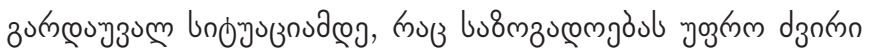

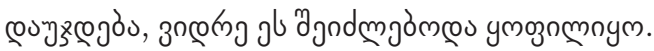

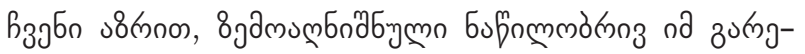

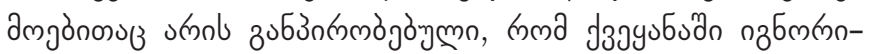

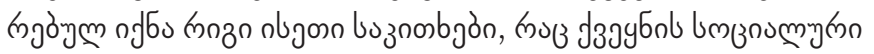

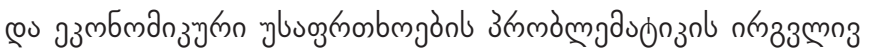

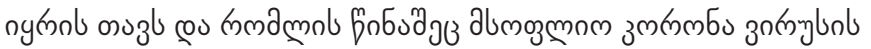

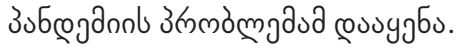

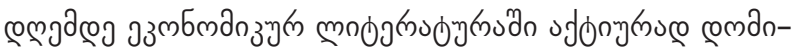

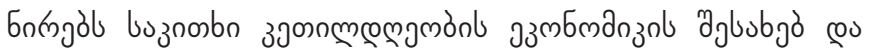

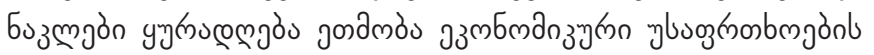

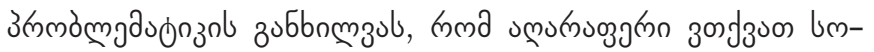

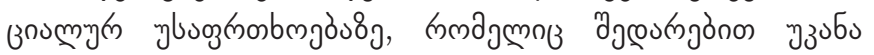

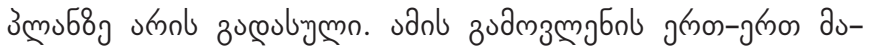

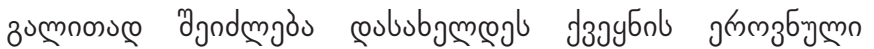

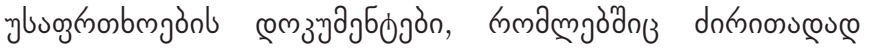

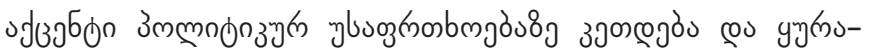

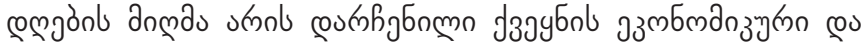

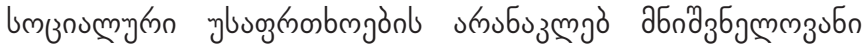

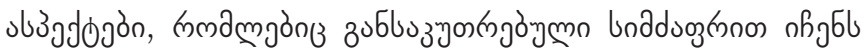

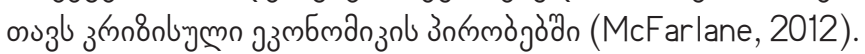

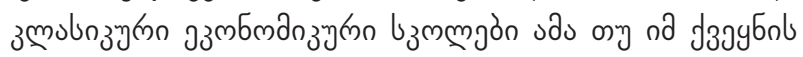

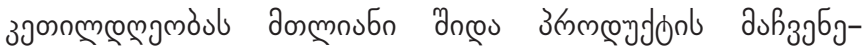

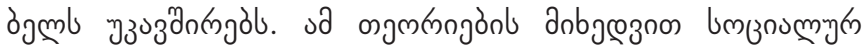

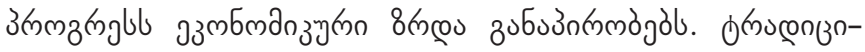

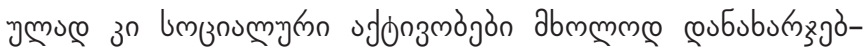

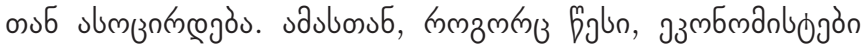

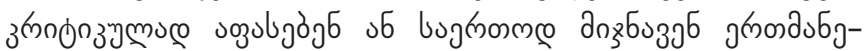

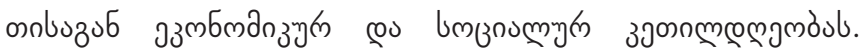

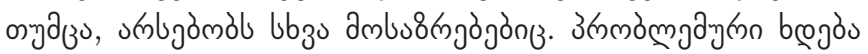

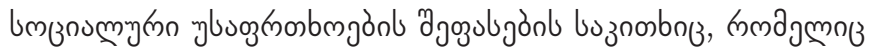

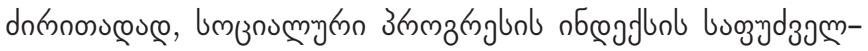

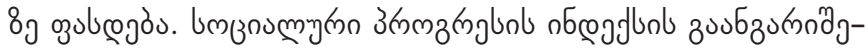

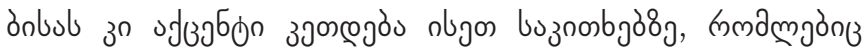

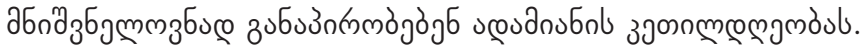

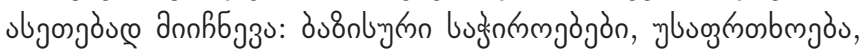

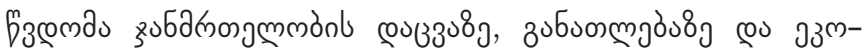

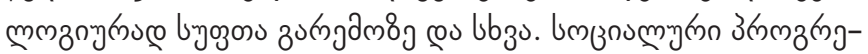

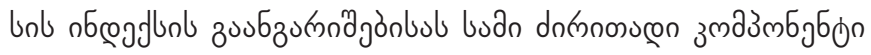

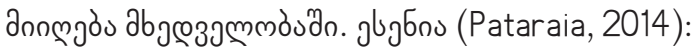

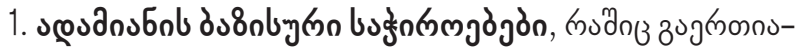

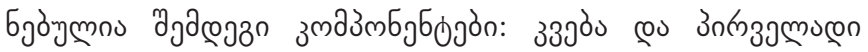

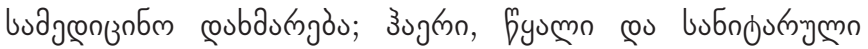

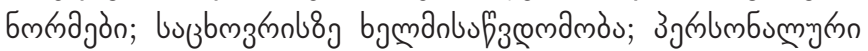
ybugुnorbmojo;

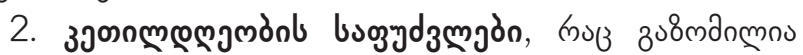

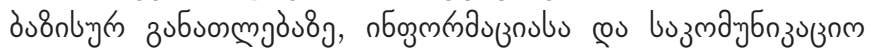

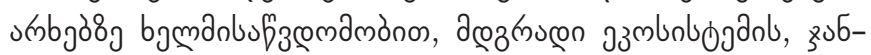

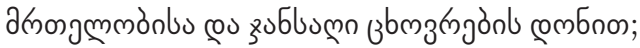

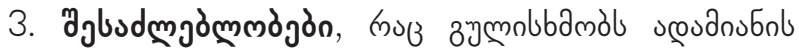

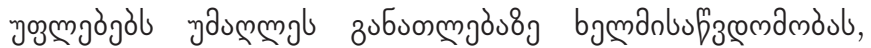
s nh bunnommmól.

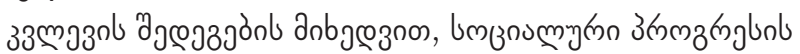

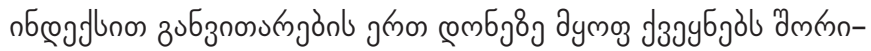

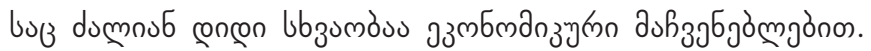

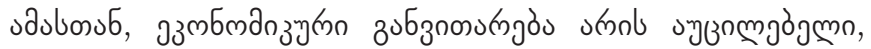

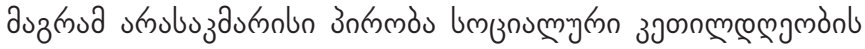

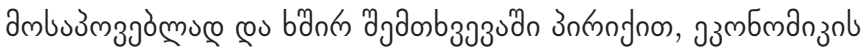

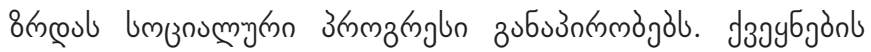

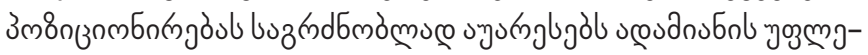

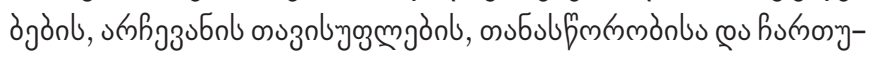
mmònl comby (Pataraia, 2014).

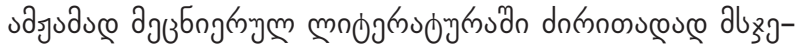

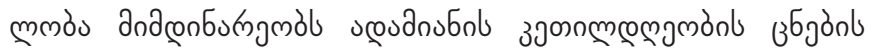

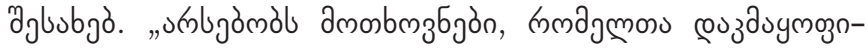

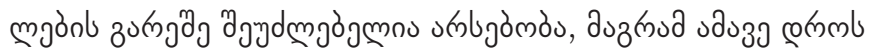




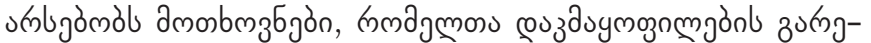

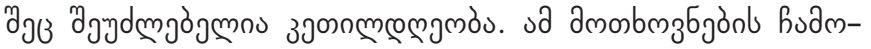

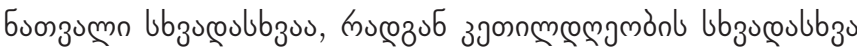

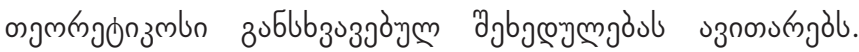

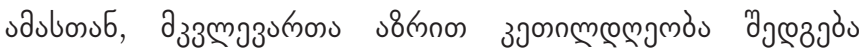

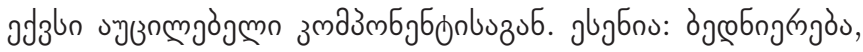

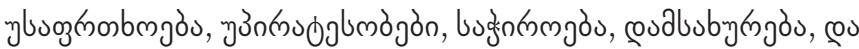

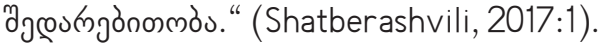

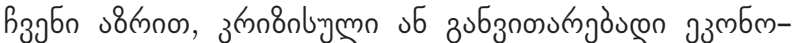

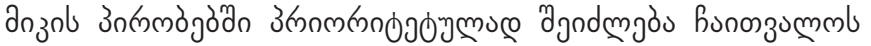

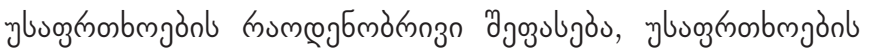

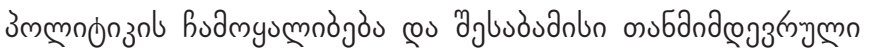

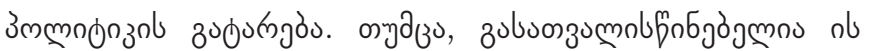

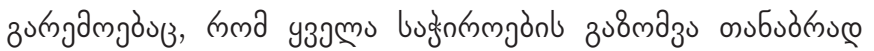

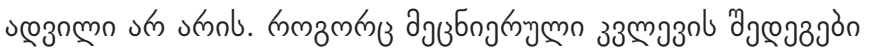

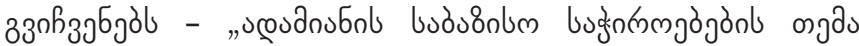

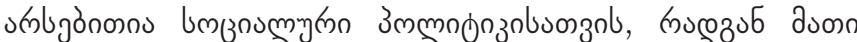

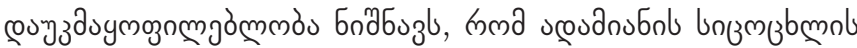

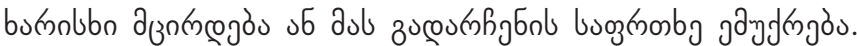

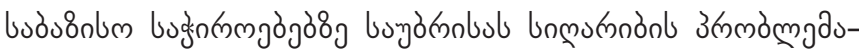

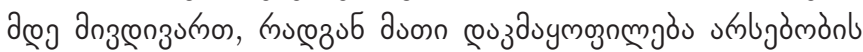

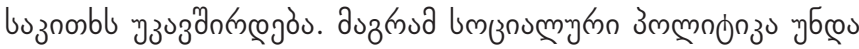

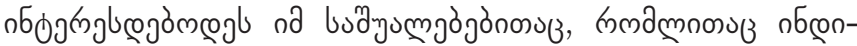

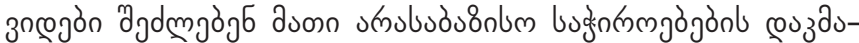

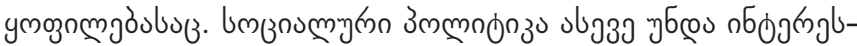

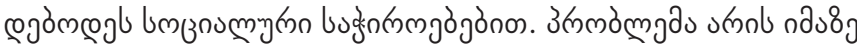

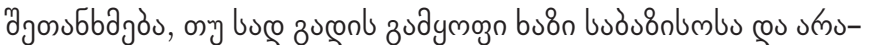

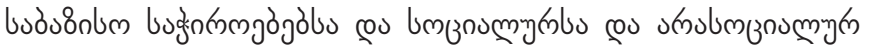

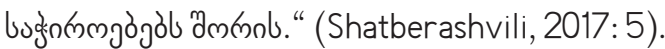

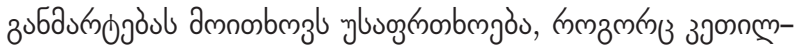

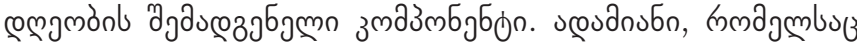

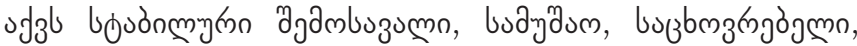

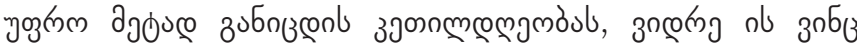

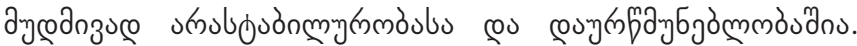

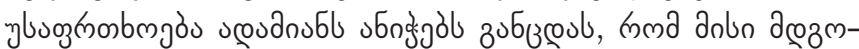

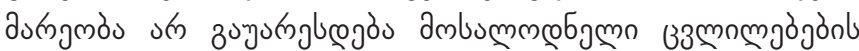

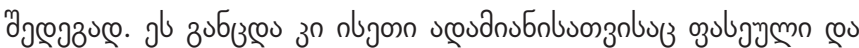

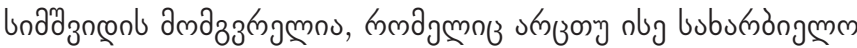

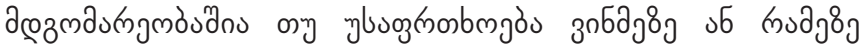

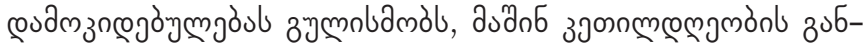

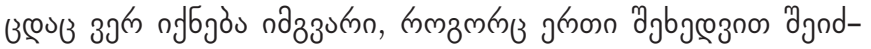
mgò $\partial m 33 \rho h 3 g b \mathrm{ml}$. (Rules of the game, 2009).

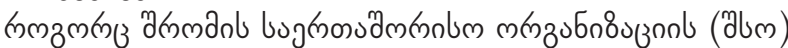

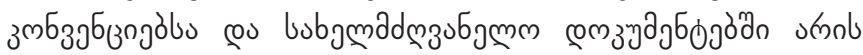

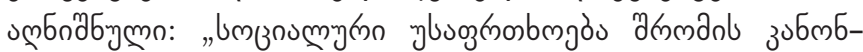

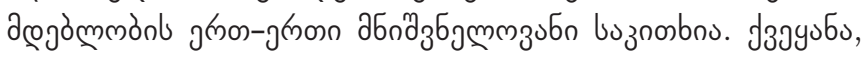

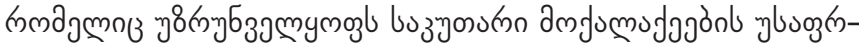

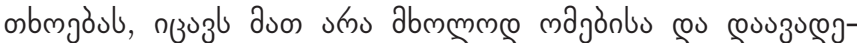

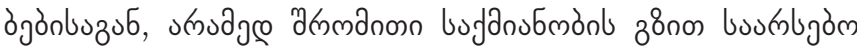

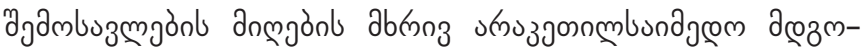

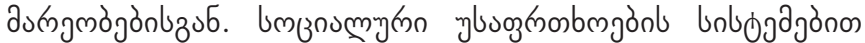

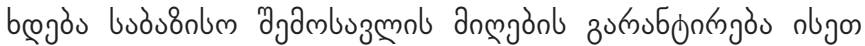

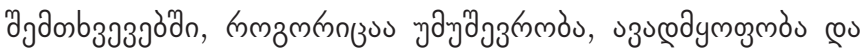

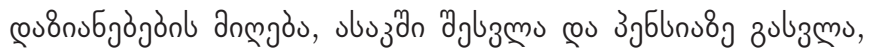

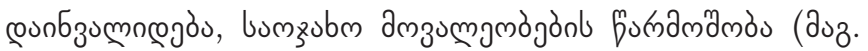

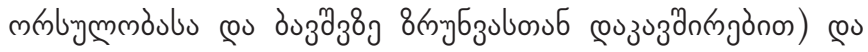

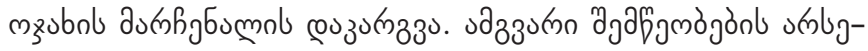

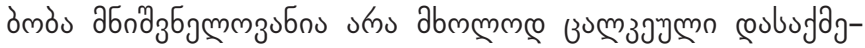

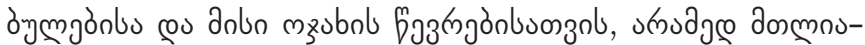

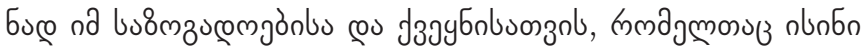

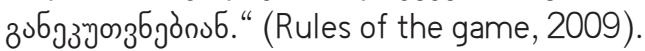

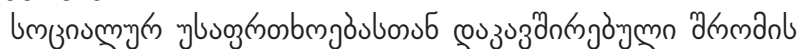

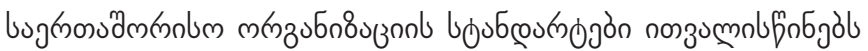

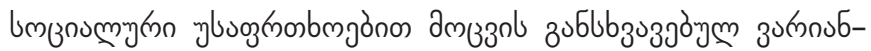

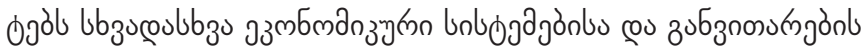

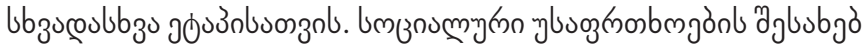

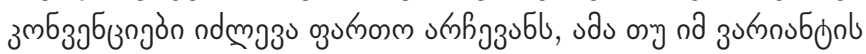

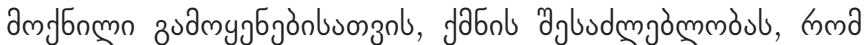

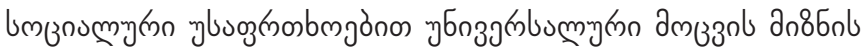

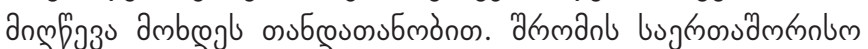

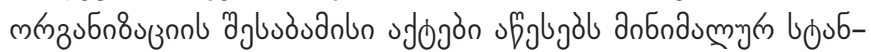

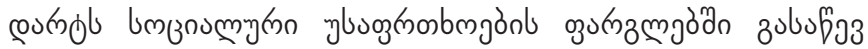

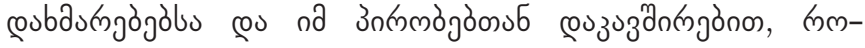

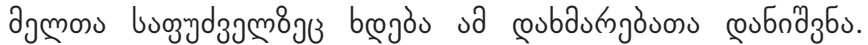

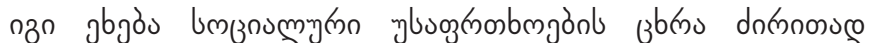

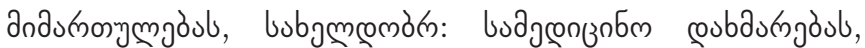

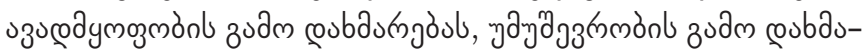

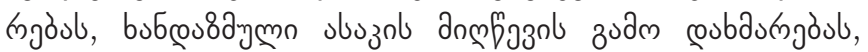

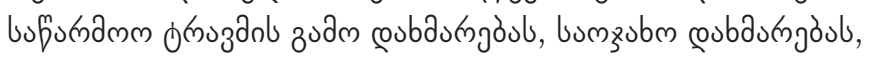

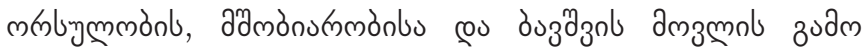

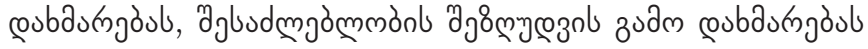

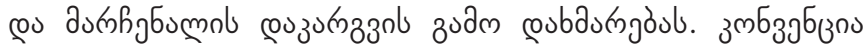

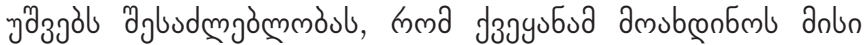

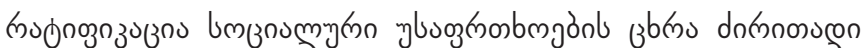

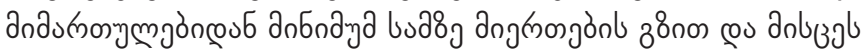

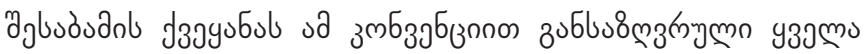

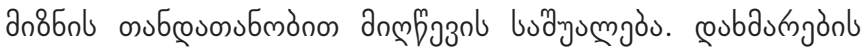

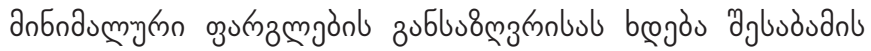

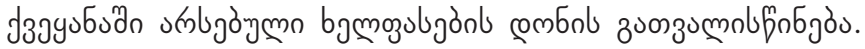

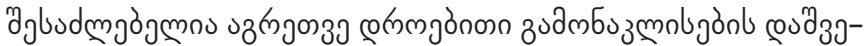

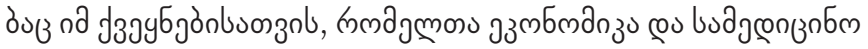

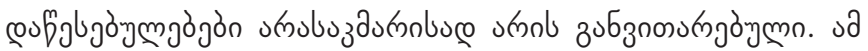

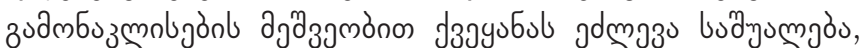

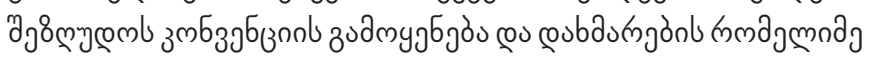

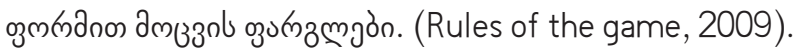

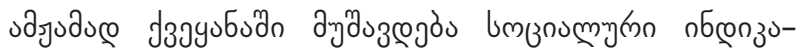

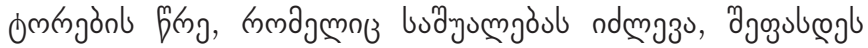

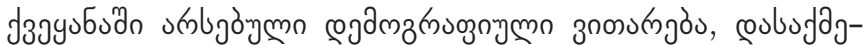

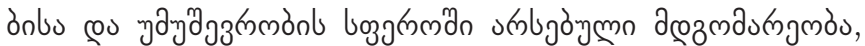

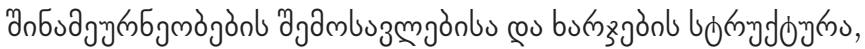




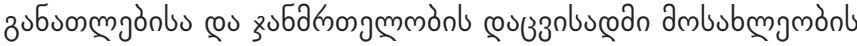

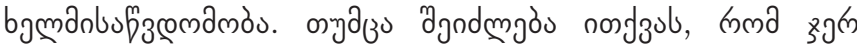

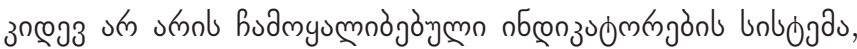

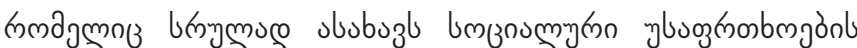

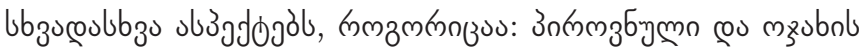

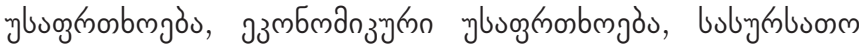

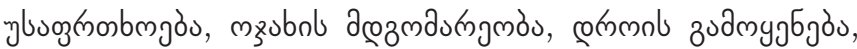

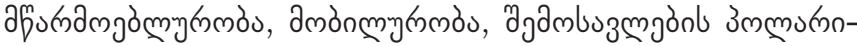

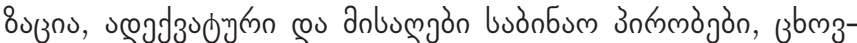

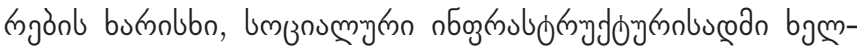

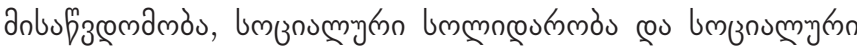

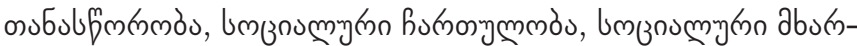

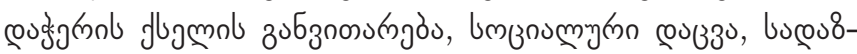

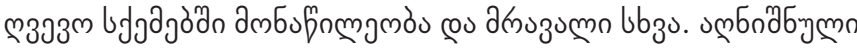

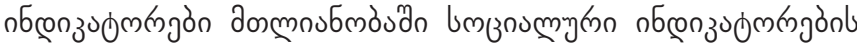

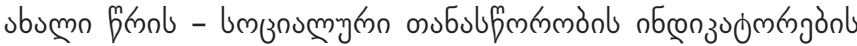

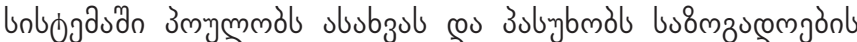

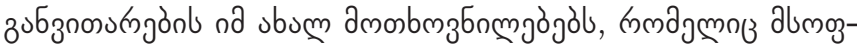

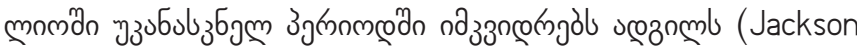
and others, 2000).

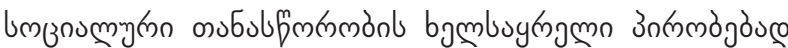

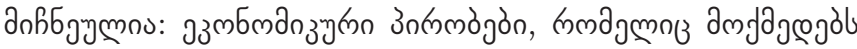

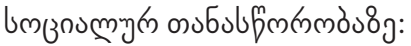

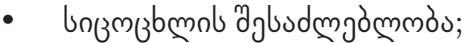

- unzmozrmol burnolibo;

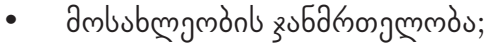

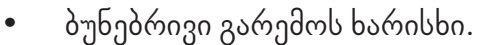

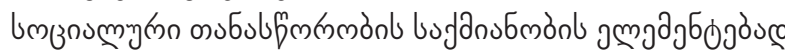

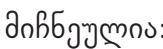

- dmbufongmoso;

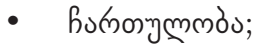

- उobsongajo

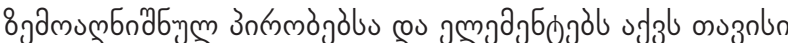

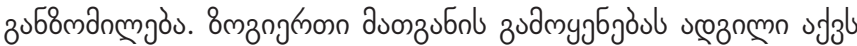

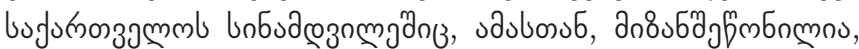

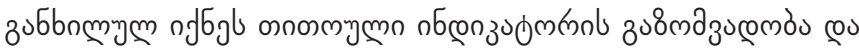

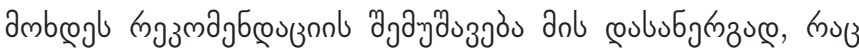

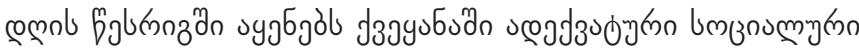

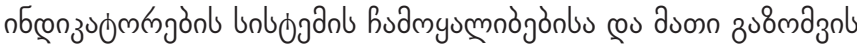

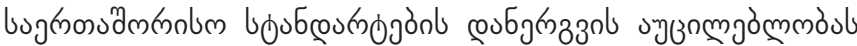
(Chikovani, 2012).

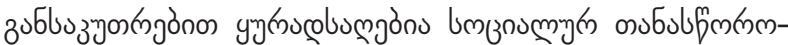

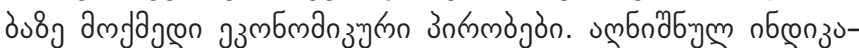

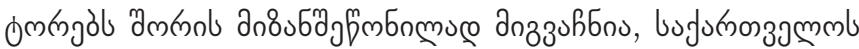

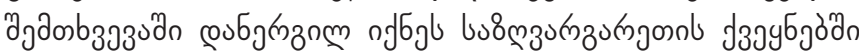

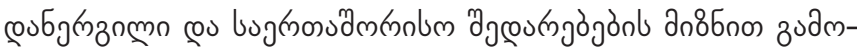

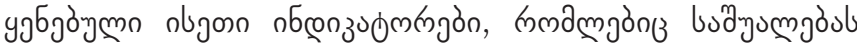

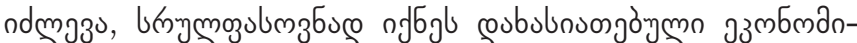

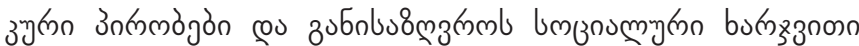

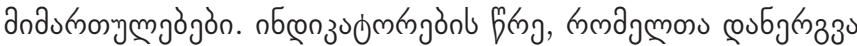

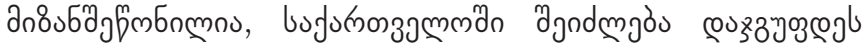

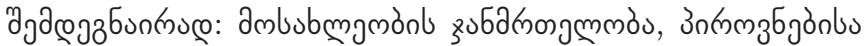

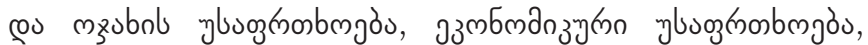

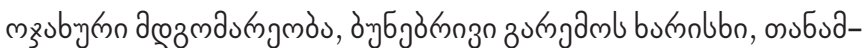

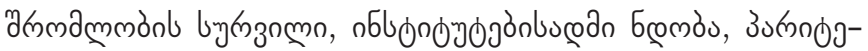

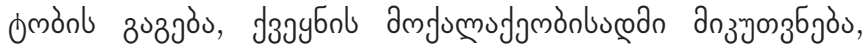

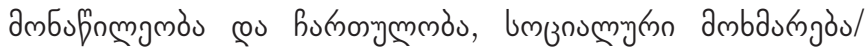

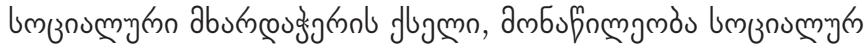

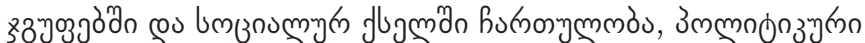

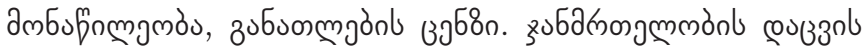

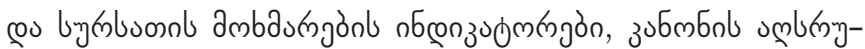

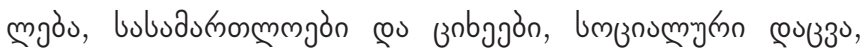

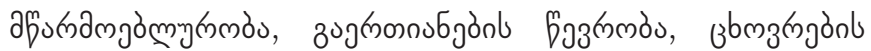

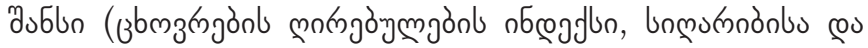

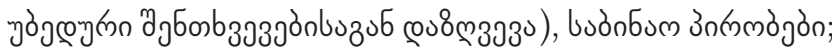

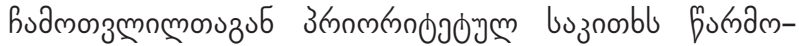

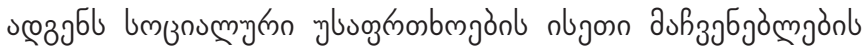

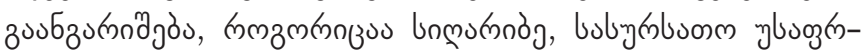

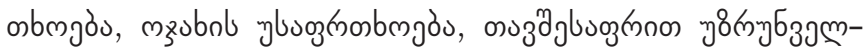

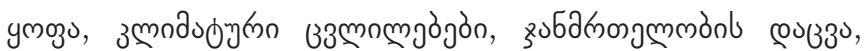

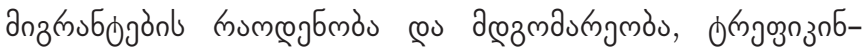

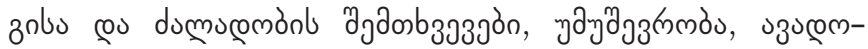

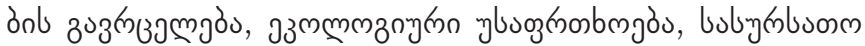

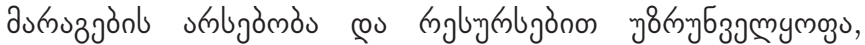

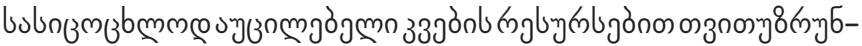

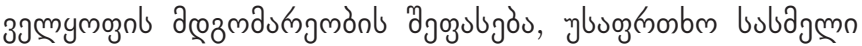

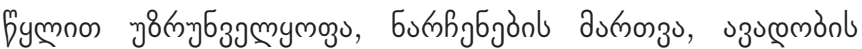

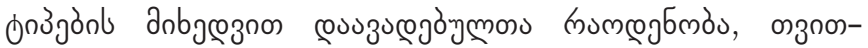

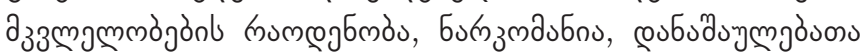

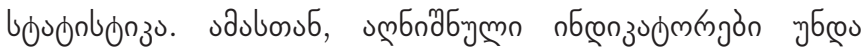

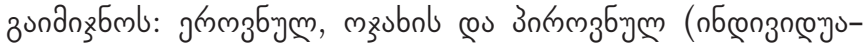

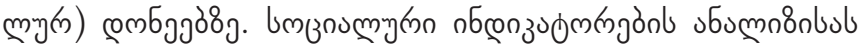

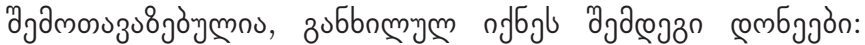

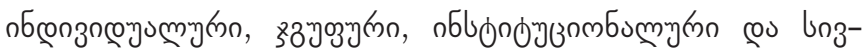

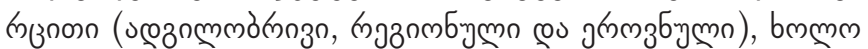

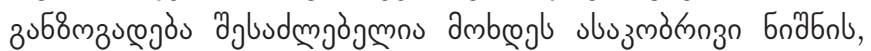

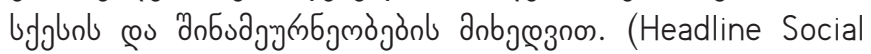
Indicators, 2009).

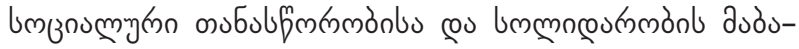

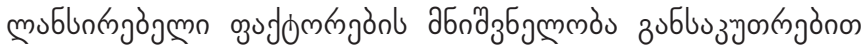

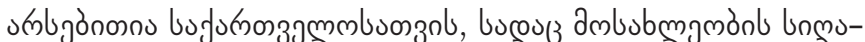

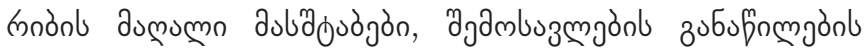

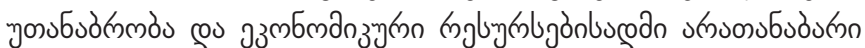

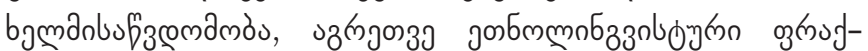

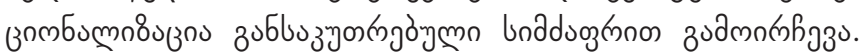

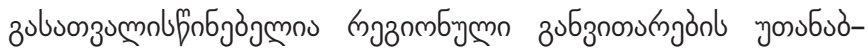

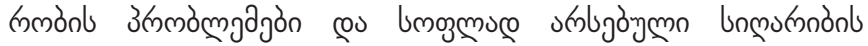

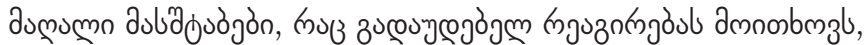

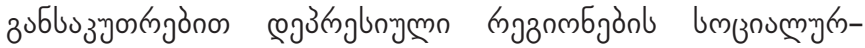

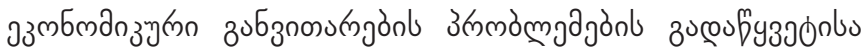




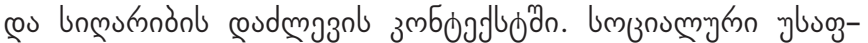

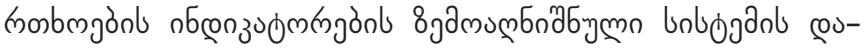

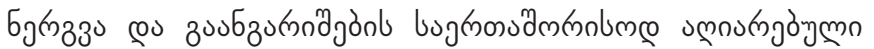

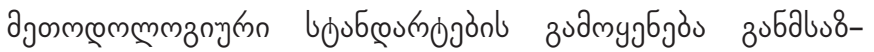

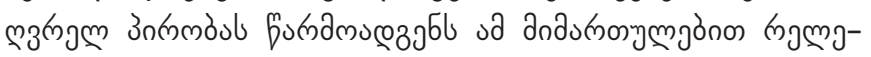

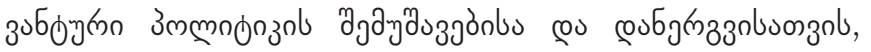

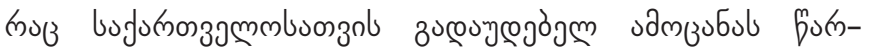

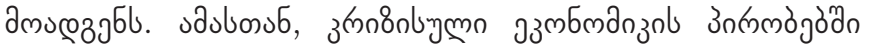

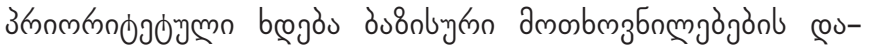

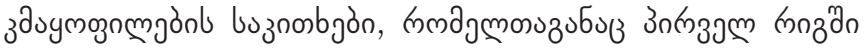

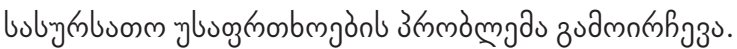

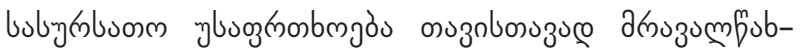

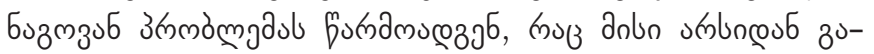

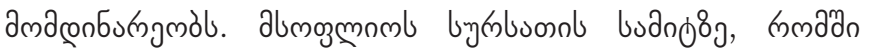

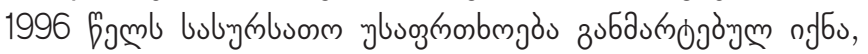

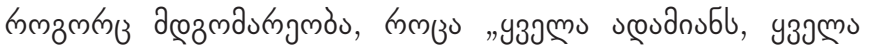

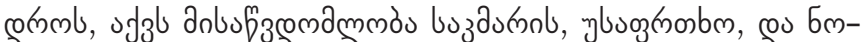
yngk, yguosnub, by

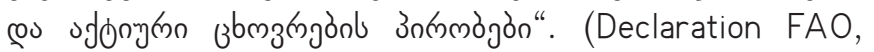

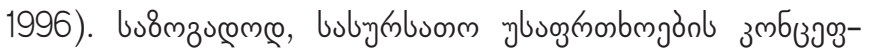

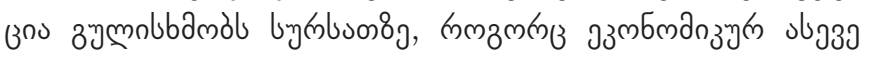

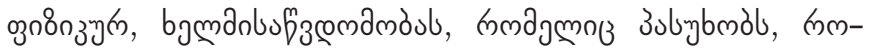

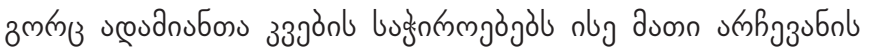

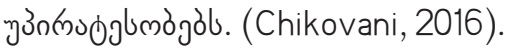

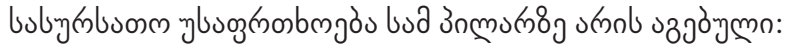

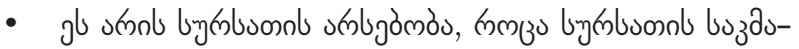

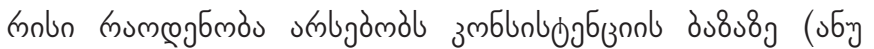

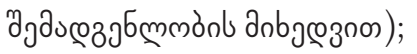

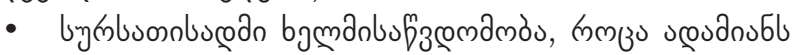

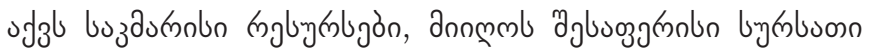
Emyngmo engonobuoszol;

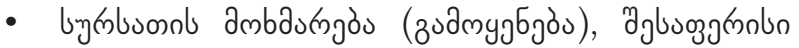

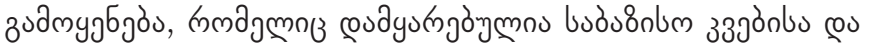

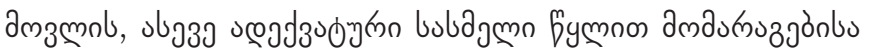

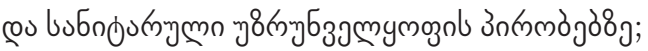

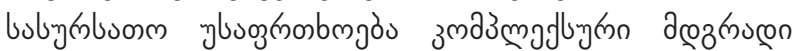

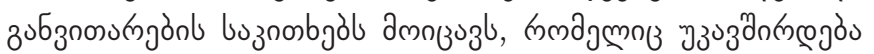

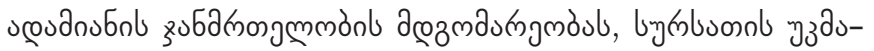

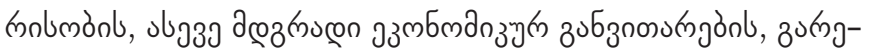

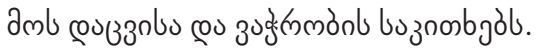

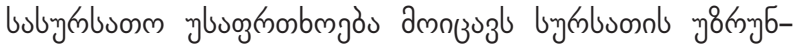

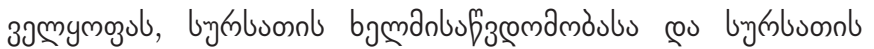

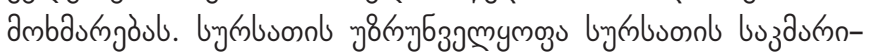

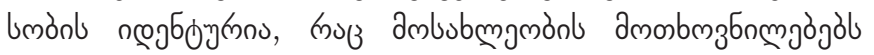

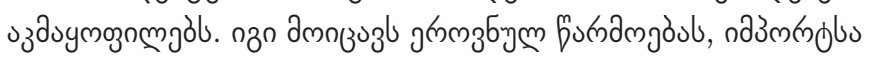
co dumozjals.

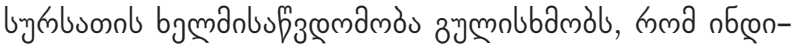

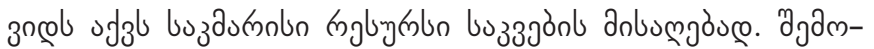

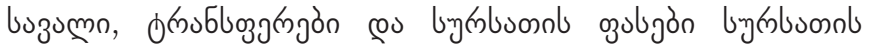

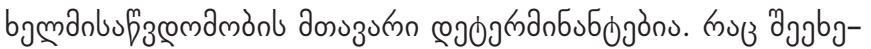

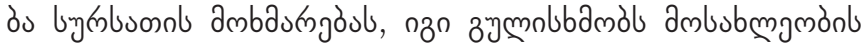

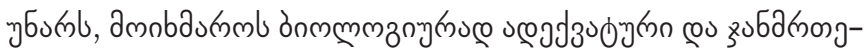

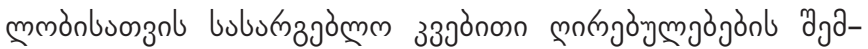

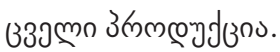

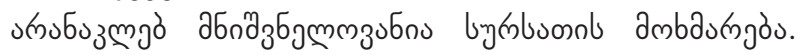

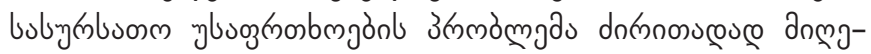

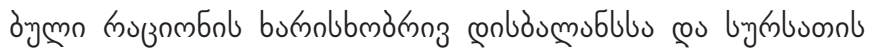

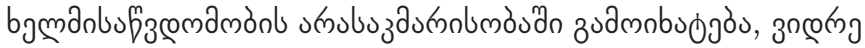

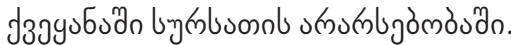

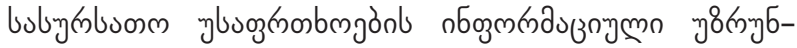

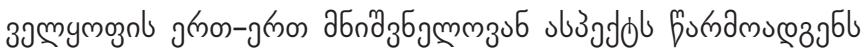

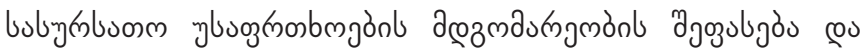

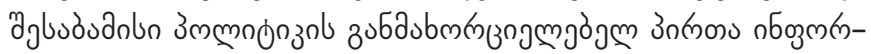

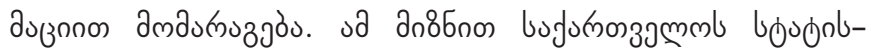

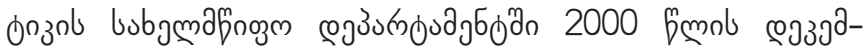

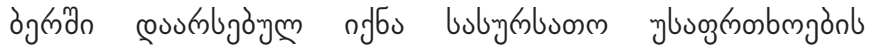

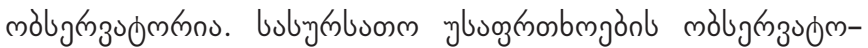

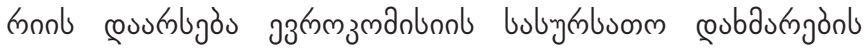

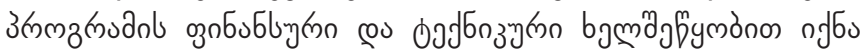

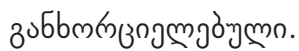

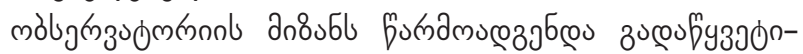

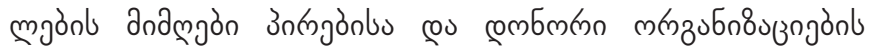

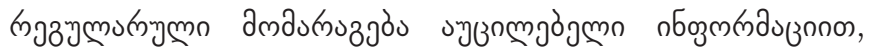

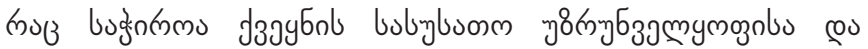

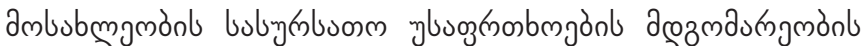
ajogubjönlus es ambnom

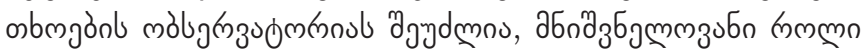

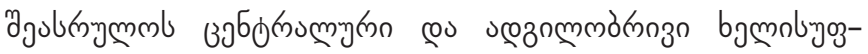

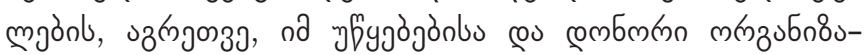

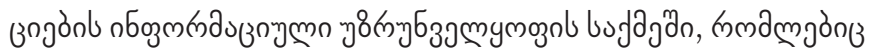

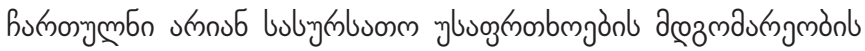

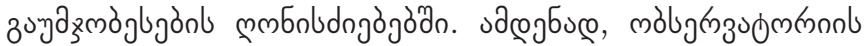

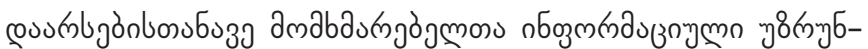

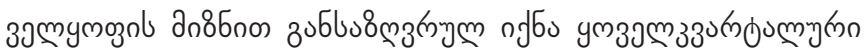

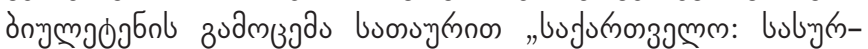

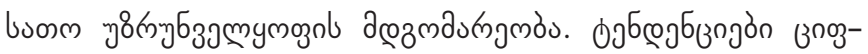

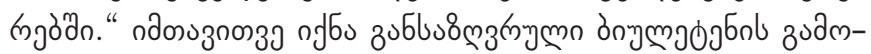

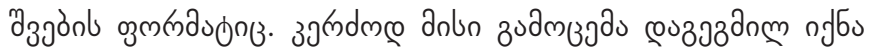

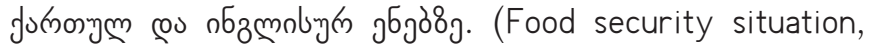

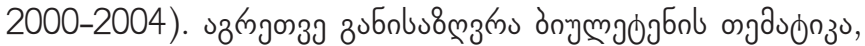

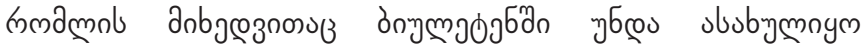
cos unbubu zn@

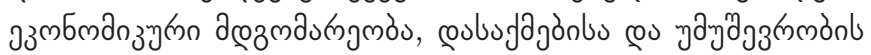

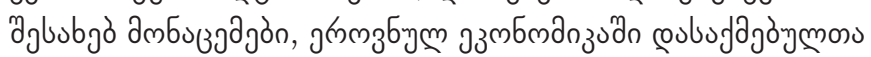

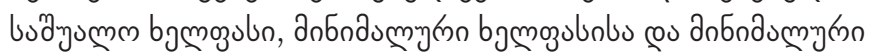

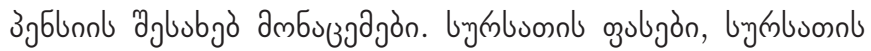

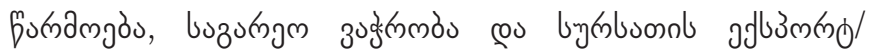

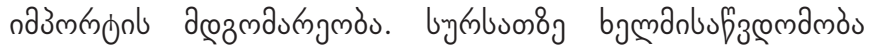

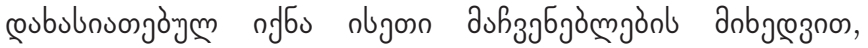

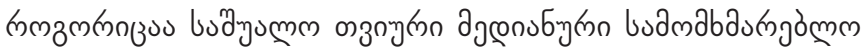




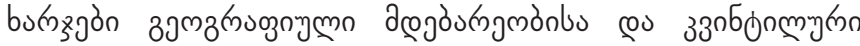

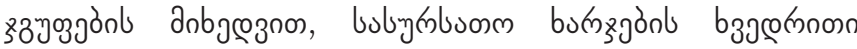

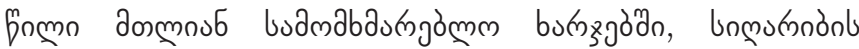

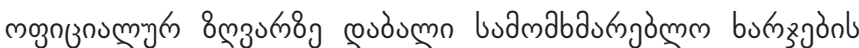

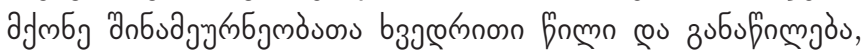

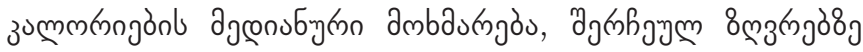

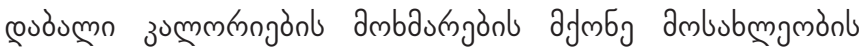

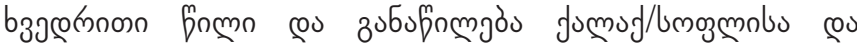

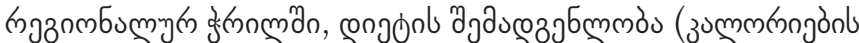

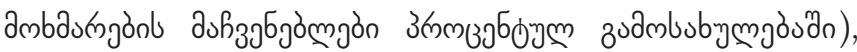

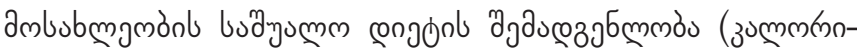

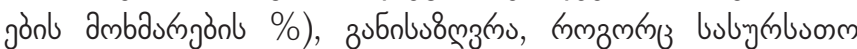

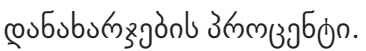

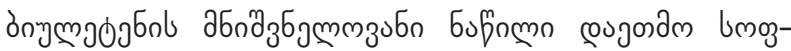

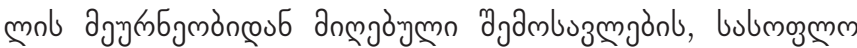

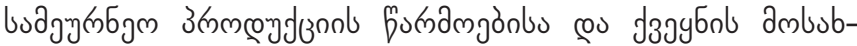

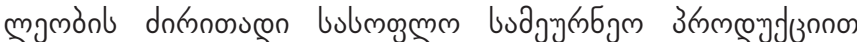

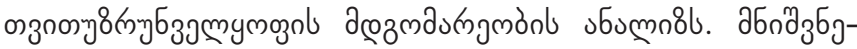

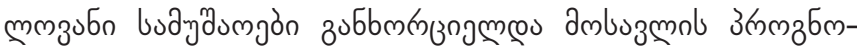

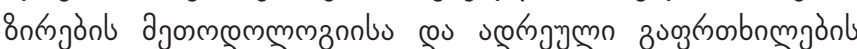

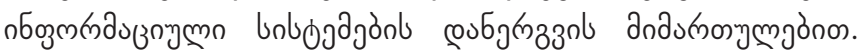

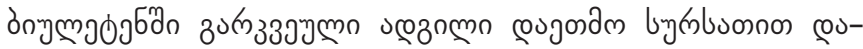

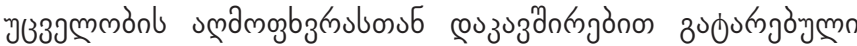

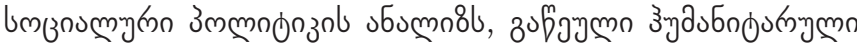

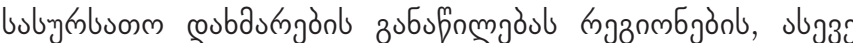

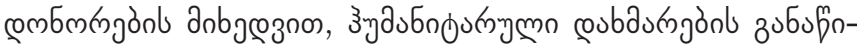

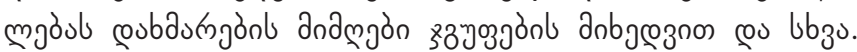

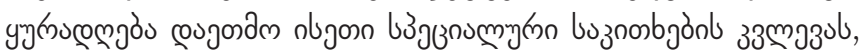

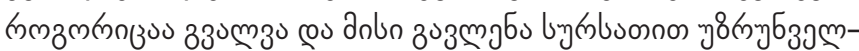
ymoु

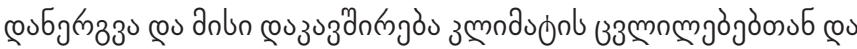

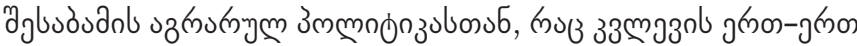

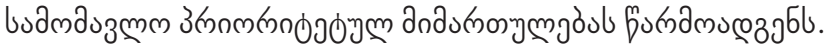

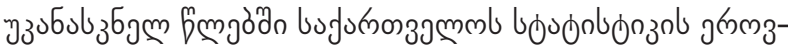

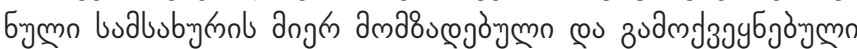

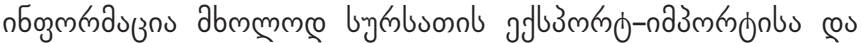

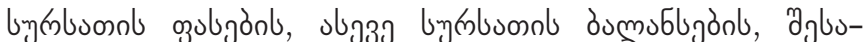

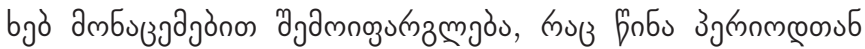

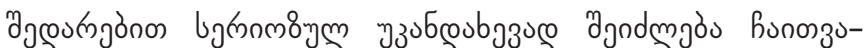

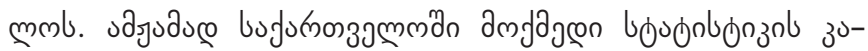

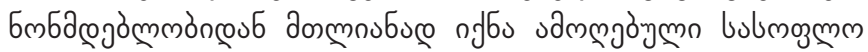

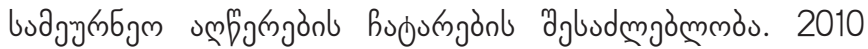

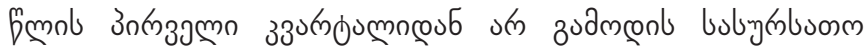

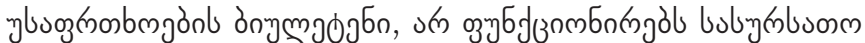

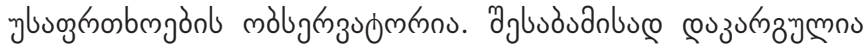

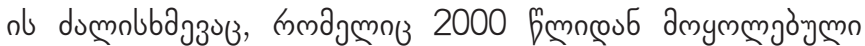

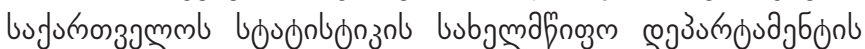

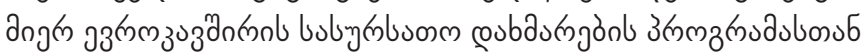

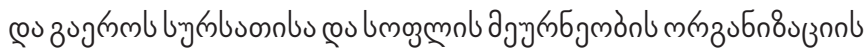

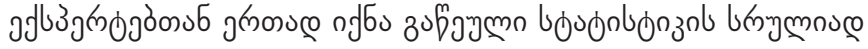

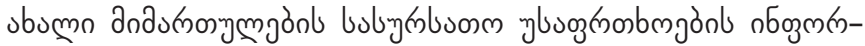

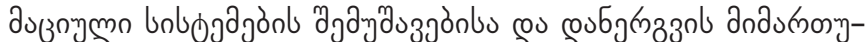

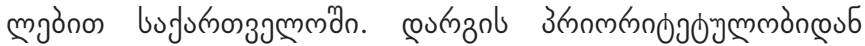

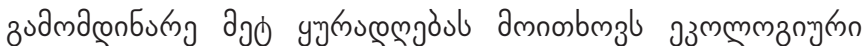

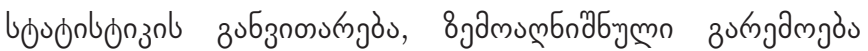

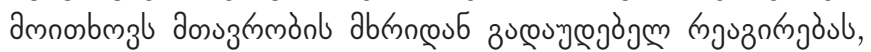

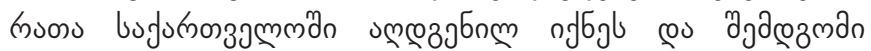

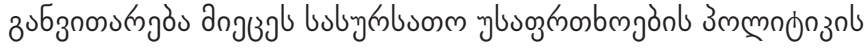

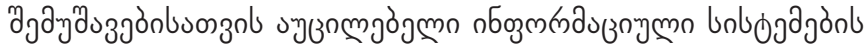

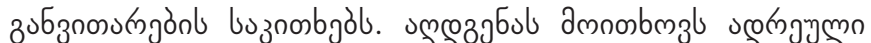

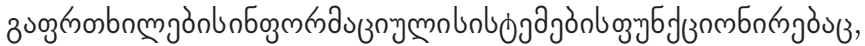

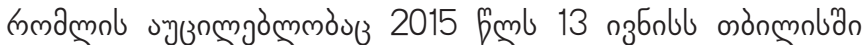

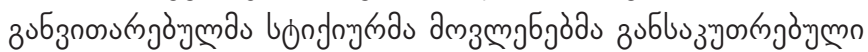

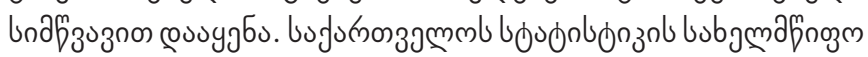

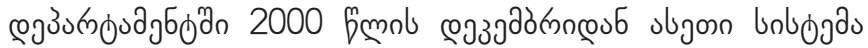

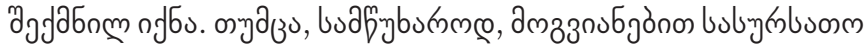

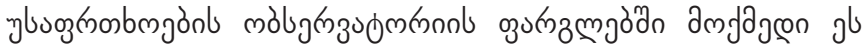

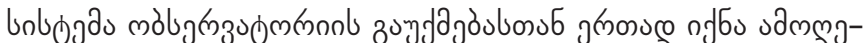

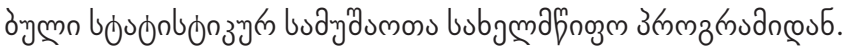

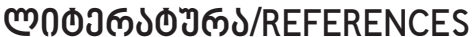

Vision for the development of labor and social protection in Georgia by 2030. European Union for Georgia. The parliament of Georgia. Tbilisi 2017; (In Georgian)

The Socio-Economic Development Strategy of Georgia; Georgia. 2020; (In Georgian)

McFarlane, N. S., Russia and Eurasia Program, Program Article 2012/01 Georgia: National Security Concept and National Security Centre for Social Sciences, Tbilisi and Oxford University August, 2012; (In Georgian)

Rules of the game. A brief introduction to international labor norms. Revised edition 2009; (In Georgian)

A new strategy for social integration. A revised strategy for social integration. Approved by the Committee of Ministers of the Council of Europe on 31 March 2004. European Committee for Social Integration (CDCS). Translation, Tb. 2007; (In Georgian)

Pataraia, L., Economic Growth and Social Prosperity. March, 2014. Forbes Opinion; (In Georgian);

Shatberashvili N., International Social Welfare. 2016 - 2017 academic year; (In Georgian)

Social Protection And Social Inclusion in Georgia. Concluding Remarks. European Commission. General Directorate of Employment, Social Affairs and Inclusion. The document was completed in 2011; (In Georgian) 
Georgia: The State of Food Security. Trends in Numbers. Statistical Bulletin, N1-19, Publishing House of the State Department of Statistics of Georgia. (In Georgian and English) Tbilisi, 2000-2005. Editor E. Chikovani; (In Georgian)

Chikovani, E., For Some Issues of Improving the System of Social Indicators in Georgia. Collection of scientific works of Tbilisi Open Teaching University. Tbilisi, 2012. 119-131; (In Georgian)

Chikovani, E., On the Functioning of Food Safety Information Systems in Georgia. Abstracts of reports. Business Development in The Global Economy. Georgian Business Academy. Proceedings of the International Scientific-Practical Conference. Tbilisi 19-20 May 2016. 118-124; (In Georgian)

Declaration FAO (1996). Rome declaration on world food security. World Food Summit, Rome, 13-17 November 1996. FAO, Rome. Headline Social Indicators. ISBN 978-92-64-04938-3 OECD Social Indicators. Society at a Glance 2009 @ OECD 2009 http:// www.oecd.org/dataoecd/29/5/42670071.pdf

Social Cohesion in Canada: Possible Indicators. Highlights by Canadian Council on Social Development Andrew Jackson, Gail Fawcet, Anne Milan, Paul Roberts, Sylvain Schetagne, Katherine Scott, Spy Tsoukalas, For Social Cohesion Network. Department of Canadian Heritage, Department of Justice. November 2000, 25 Eddy street, Quebec, Canada

Social Cohesion: The Canadian Urban Context. Prepared by: Michael Toye, 25 October 2007; PDF (106 Kb, 16) 